\title{
Vogt-Koyanagi-Harada Disease and Sympathetic Ophthalmia: Is there an Immune Mediated Etiopathologic Relationship with Neural Crest Cell Derivatives and Ciliary Body Stem Cells?
}

\author{
Pradeep Venkatesh* \\ Dr. Rajendra Prasad Centre for Ophthalmic Sciences, All India Institute of Medical Sciences, New Delhi, India
}

*Corresponding author: Pradeep Venkatesh, Dr. Rajendra Prasad Centre for Ophthalmic Sciences, E-104, AllMS campus, Ansari Nagar, New Delhi-110029, India, Tel: 011-2658-9695; E-mail: venkyprao@yahoo.com

Received date: February 19, 2016; Accepted date: March 26, 2016; Published date: April 02, 2016

Copyright: (2016 Venkatesh P. This is an open-access article distributed under the terms of the Creative Commons Attribution License, which permits unrestricted use, distribution, and reproduction in any medium, provided the original author and source are credited.

\section{Abstract}

Vogt Koyanagi Harada disease (VKH) is a multisystem disorder of an unknown, possibly immune etiology. Sympathetic ophthalmia is also a bilateral panuveitis of presumed immune origin where the inciting stimulus is an injury to the eyeball. The etiopathogenesis of these two diseases which manifest as panuveitis remains poorly understood. In this review, an effort is made to draw a possible etiological association between ciliary body stem/ progenitor cells and sympathetic ophthalmia and one between Vogt-Koyanagi-Harada disease and neural derived crest cells.

Keywords: Sympatheic ophthalmia; VKH syndrome; Stem cells; Ciliary body stem cells; Neural crest cells; Autoimmune

\section{Introduction}

Vogt Koyanagi Harada disease (VKH) is a multisystem disorder of an unknown, possibly immune etiology characterized by ocular, cutaneous, neurological and auditory features [1]. It is also known as uveomeningeal syndrome. In the eye, it presents as a bilateral panuveitis of sudden onset and results in a fairly rapid decrease in vision if there is a delay in diagnosis and treatment. Cutaneous features include alopecia and vitiligo, neurological features are meningeal irritation, cranial nerve palsies, pleocytosis on cerebrospinal fluid analysis and convulsions and auditory symptoms, transient vertigo, tinnitus and loss of hearing. The disease normally progresses through the prodromal stage, ocular stage, chronic stage and recurrent stage. Ocular inflammation generally precedes the integumentary changes but may also occur concurrently. The mainstay of treatment is immunomodulatory therapy with corticosteroids and immunosuppressives (azathioprine, methotrexate, cyclosporine, mycophenolate etc.).

Sympathetic ophthalmia is again a bilateral panuveitis of presumed immune origin where the inciting stimulus is a major injury to the eyeball. The injury in the majority of patients involves uveal tissue (iris, ciliary body and choroid) so much so that the ciliary body is considered a danger zone for the development of sympathetic ophthalmia. Not only trauma, but even a minor or major surgical procedure that disturbs the uveal tissue can incite sympathetic ophthalmia. The eye which sustains the injury is called the exciting eye and the fellow eye which becomes inflamed secondarily, as the sympathizing eye. Involvement of the fellow eye may occur within 10 days of sustain the injury to several years (with $90 \%$ of cases developing within the first year) [2]. The risk of developing sympathetic ophthalmia is highest when the penetrating trauma involves the ciliary body (danger zone). Like VKH, there is high risk of visual loss if the condition is not diagnosed and treated early [3]. As in the case of $\mathrm{VKH}$, treatment revolves around the use of corticosteroids and immunosuppressives $[4,5]$.

Sympathetic ophthalmia and VKH have been known for several centuries and are characterized by the above well recognized clinical features. However, at the cellular and molecular level they remain poorly understood and their etiopathogenesis has remained an enigma [6-8]. With this background, the current article tries to explore the possible relationship between sympathetic ophthalmia and the recently detected ciliary body stem cells. The second relationship it presents is that between Vogt-Koyanagi-Harada disease and neural crest cells.

\section{Current understanding}

How injury to one eye incites inflammation in the fellow eye continues to remain an enigma. For over a century, it has been speculated that sympathetic ophthalmia is an immune mediated pathology with uveal pigment (melanocytes) being the possible antigenic stimulus [9]. However studies have shown that cell mediated immunity in sympathetic ophthalmia is not only directed against uveal antigen but also against retinal antigens found on photoreceptors and retinal pigment epithelial cells $[10,11]$. The retina being an immune privileged site and the choroid lacking in lymphatics, it is difficult to conjecture as to how any antigen would produce a sympathizing response in the fellow eye. To explain this some authors proposed that following injury to the ocular coats, the spread of antigens occurs through conjunctival lymphatic 
vessels [12]. The sensitizing reaction then occurs in the regional lymph nodes and spleen. Another suggestion has been that a transient antigen-antibody reaction is induced by exposure to high dose of antigen in the immediate posttraumatic period. Hence the current opinion is that there are several retinal and uveal antigens against which autoimmunity and cell mediated immunity is initiated in patients with sympathetic ophthalmia. However the source of these antigens still remains unknown. In this context it is also important to emphasize that not all patients with significant penetrating trauma develop sympathetic ophthalmia. The prevalence being 1 in 5000 to 1 in 2000 patients with ocular trauma and 1 in 10,000 patients following intraocular surgery $[13,14]$. So, the sympathizing response is not universal. As mentioned in the introduction, one established observation has been that injury to the ciliary region is considered 'danger zone' for the development of sympathetic ophthalmia. Why this is so also remains unexplained. How these issues could be explained by using the putative source of antigen as being the ciliary body stem cells is discussed in the subsequent section.

As regards $\mathrm{VKH}$, the etiology still remains unanswered. A viral infection in a genetically predisposed person setting off an autoimmune reaction against melanocytes is the most considered etiology presently [15]. Studies showing close contact between lymphocytes and melanocytes, serum containing lymphocytes with cytotoxic activity against B-36 melanoma cell line and IL-2 dependent T cells targeting normal melanocytes and melanoma cells support this melanocyte theory $[16,17]$. Melanocyte associated peptides related to tyrosinase, TRP-1 and TRP-2 have been shown to be antigenic in intraocular fluids [18]. Autoantibodies against Mueller cells and photoreceptor outer segments have also been detected in the sera [19]. These peptides have also been studied in an experimental model of VKH [20]. High titres against an antigen expressed by melanocytes, KU-MEL-1 have also been demonstrated in patients with VKH [21]. These studies highlight the possibility of melanocytes being the primary antigenic stimulus in this disease. However they fail to address other immune observations like the presence of antibodies against retinal $\mathrm{S}$ antigen, interphotoreceptor retinoid binding protein (IRBP), photoreceptors and Muller cells remains unexplained. Most importantly however, this theory again fails to explain the other multisystem components of VKH like deafness, meningeal signs, cranial nerve palsies and auditory features (deafness, vertigo and tinnitus). Also, the presence of a viral infection is frequently lacking to support the consideration of post-viral autoimmune mediated disease. In the next section an effort is made to raise the possibility of neural crest derived cells being a strong contender in the pathogenesis of VKH.

\section{Ciliary body stem cells and neural crest derived cells}

In fish and amphibians, it has been proven that the retina continues to grow throughout life and is also able to regenerate. This has been attributed to the presence of stem cells in the ciliary marginal zone [22]. With evolution of species, this zone is said to have progressively regressed and almost become non-existent in mammals, including humans [23]. However in the year 2000, it was postulated that the ciliary body (the midzone of the uveal layer between the anterior iris and posterior choroid) may harbor retinal stem cells $[24,25]$. Although the mammalian ciliary body is largely composed of small muscular tissue, the epithelium which lines it has neuroepithelial features and shares a common origin with the optic cup during embryonic development. Two research teams independently reported that single pigmented cells from the mouse ciliary epithelium could clonally proliferate and had the ability of self-renewal. Interestingly, these cells within the colony were shown to express genes found in photoreceptors, bipolar cells and Mueller cells. Subsequently, similar ciliary body stem cells were reported in humans too. These stem cells also have only a limited ability of proliferation and self-renewal. More recently several authors have questioned the presence of true neural stem cells in the human ciliary body. The conclusion from a current review states that although true neural stem cells are absent from the human ciliary body, there certainly exists a population of cells with remarkable plasticity and characteristics of neuroepithelial progenitors (and their markers) [26]. It has also been shown that they can differentiate into rhodopsin positive cells in specific vitro conditions [27]. The retinal and neural markers expressed by these progenitor cells include Nestin, Lhx2, Dach1, Chx10, Six 3, Sox 2 and Pax6 [28]. The number of ciliary body epithelial cells with these progenitor properties is considered to be only $0.2 \%$ to $2 \%[25,28,29]$.

During the formation of the neural tube (which finally develops into the spinal cord), some neural embryonic cells get separated and no longer remain as a component of the central nervous system. These cells, called neural crest cells migrate to various regions of the body and transform into several cell derivatives. Some of neural crest cell derivatives include melanocytes, odontoblasts, meningeal covering of the brain, Schwann cells, sensory ganglia, thymic cells, sympathetic and parasympathetic ganglia, cranial neurons, facial bones and cartilage, some cells of the adrenal medulla and ossicles (bones of the middle ear-anvil, malleus and stapes) [30,31]. In the eye neural crest cell derivatives include choroidal melanocytes. Owing to their ability to form various cellular forms (multipotency) and limited ability of self-renewal, neural crest cells are considered to bear the hallmarks of stem cells and progenitor cells. Markers for pluripotency, NANOG, POU5FI and SOX2 have also been found to be expressed on neural crest cells further strengthening their ability to behave like stem cells $[32,33]$. It is however likely that similar to ciliary body cells, not all adult neural crest cells possess the capabilities of stem cells. There could be only a small fraction of these cells still retaining stem cell/ progenitor cell property.

\section{Possible etiopathological relationship}

Sympathetic ophthalmia and ciliary body stem cells/ progenitors: The possibility of ciliary body stem cells/ progenitors being the antigenic source to incite sympathetic ophthalmia is supported by the following clinical observations- 
Not all patients with intraocular trauma develop sympathetic ophthalmia. The prevalence being 0.01\%-0.05\%. This can be explained by studies which show that the number of ciliary body epithelial cells with progenitor properties is only $0.2 \%$ to $2 \%$. Hence, even if the ciliary 'danger' zone is involved in the trauma, sympathetic ophthalmia is likely to develop only in those patients in whom ciliary body progenitor cells get activated and become antigenic.

In addition to uveal antigen, those against retinal photoreceptors have also been consistently demonstrated in patients with sympathetic ophthalmia. This is explainable by reports which demonstrate that ciliary body progenitor cells also express markers for photoreceptors, bipolar cells and Mueller cells.

Vogt-Koyanagi-Harada disease and neural crest cell derivatives: As mentioned early on, $\mathrm{VKH}$ is a multisystem disease involving ocular, cutaneous, neurological and auditory organs. While the ocular and cutaneous features (alopecia and vitiligo) can be explained with the melanocyte as the antigenic source, the other two, neurological and auditory features cannot be accounted for. If however one considers the neural crest derived cells to be the primary antigenic stimulus or cells that become antigenic secondary to antigenic conversion of melanocytes, then we could explain the neurological and auditory features. Neurological features are explained the neural crest origin of meningeal covering, Schwann cells and cranial ganglia. Auditory signs could be explained by the neural crest origin of the three ossicles and glial cells of the cochleavestibular nerve in the inner ear.

If indeed neural crest derived cells happen to be the antigenic stimulus for the development of $\mathrm{VKH}$, it raises the possibility that injury to any of its derivatives may incite the condition. VKH is not very common and varies in prevalence various populations. Other than genetic reasons this could also be related to the percent of neural crest cells that actually retain the property of stem cell/ progenitor cell into adulthood.

\section{Conclusion}

Stem cells have been widely studied for their ability to differentiate into several cells lines and self-renewal capability. So concerns with the therapeutic use of stem cells has been the risk of oncogenecity and genetic instability. After birth, it is also possible that under certain conditions (e.g. injury) residual cells with stem cell/ progenitor cell properties become exposed and turn antigenic. This initiates an immune cascade against all cells carrying similar antigens. I have herein discussed such a possible relationship in disease pathogenesis with regards to two autoimmune disorders of the eye, the first being the relationship between sympathetic ophthalmia and ciliary body stem cells and the second, that between VKH and neural crest derived cells. Molecular studies on patients to isolate markers for these cells and experimental studies would be useful to further improve our understanding of these two enigmatic immune mediated disorders. In the body, similar patho-mechanism may seem to contribute to other autoimmune disorders.

\section{References}

1. Moorthy RS, Inomata H, Rao NA (1995) Vogt-Koyanagi-Harada syndrome. Surv Ophthalmol 39: 265-292.

2. Lubin JR, Albert DM, Weinstein M (1980) Sixty-five years of sympathetic ophthalmia. A clinicopathologic review of 105 cases (1913-1978). Ophthalmology 87: 109-121.

3. Makley TA Jr, Azar A (1978) Sympathetic ophthalmia. A longterm follow-up. Arch Ophthalmol 96: 257-262.

4. Wand K, Straub M, Lohmann CP, Mayer CS (2016) Sympathetic ophthalmia- Therapy with steroid free immunosuppressant azathioprine. Ophthalmologe Feb 15 (E pub ahead of print). PMID: 26879557

5. Payal AR, Foster CS (2016) Long-Term Drug-Free Remission and Visual Outcomes in Sympathetic Ophthalmia. Ocul Immunol Inflamm 25: 1-6.

6. Arevalo JF, Garcia RA, Al-Dhibi HA, Sanchez JG, Suarez-Tata L (2012) Update on sympathetic ophthalmia. Middle East Afr J Ophthalmol 19: 13-21.

7. Kaneko Y, Wu GS, Saraswathy S, Vasconcelos-Santos DV, Rao NA (2012) Immunopathologic processes in sympathetic ophthalmia as signified by microRNA profiling. Invest Ophthalmol Vis Sci 53: 4197-4204.

8. Ng JY, Luk FO, Lai TY, Pang CP (2014) Influence of molecular genetics in Vogt-Koyanagi-Harada disease. J Ophthalmic Inflamm Infect 4: 20.

9. Goto H, Rao NA (1990) Sympathetic ophthalmia and VogtKoyanagi-Harada syndrome. Int Ophthalmol Clin 30: 279-285.

10. Rao NA, Robin J, Hartmann D, Sweeney JA, Marak GE Jr (1983) The role of the penetrating wound in the development of sympathetic ophthalmia experimental observations. Arch Ophthalmol 101: 102-104.

11. Jakobiec FA, Marboe CC, Knowles DM 2nd, Iwamoto T, Harrison W, et al. (1983) Human sympathetic ophthalmia. An analysis of the inflammatory infiltrate by hybridoma-monoclonal antibodies, immunochemistry, and correlative electron microscopy. Ophthalmology 90: 76-95.

12. Albert DM, Diaz-Rohena R (1989) A historical review of sympathetic ophthalmia and its epidemiology. Surv Ophthalmol 34: 1-14.

13. Du L, Kijlstra A, Yang P (2016) Vogt-Koyanagi-Harada disease: Novel insights into pathophysiology, diagnosis and treatment. Prog Retin Eye Res.

14. Marak GE Jr (1979) Recent advances in sympathetic ophthalmia. Surv Ophthalmol 24: 141-156.

15. Gomi CF, Makdissi FF, Yamamoto JH, Olivalves E (1997) An epidemiologic study on uveitis. Rev Med 76: 101-8

16. Maezawa N, Yano A, Taniguchi M, Kojima S (1982) The role of cytotoxic $\mathrm{T}$ lymphocytes in the pathogenesis of Vogt-KoyanagiHarada disease. Ophthalmologica 185: 179-186.

17. McClellan KA, MacDonald M, Hersey P, Billson FA (1989) VogtKoyanagi-Harada syndrome--isolation of cloned $T$ cells with specificity for melanocytes and melanoma cells. Aust N Z J Ophthalmol 17: 347-352. 
18. Yamaki K, Gocho K, Hayakawa K, Kondo I, Sakuragi S (2000) Tyrosinase family proteins are antigens specific to VogtKoyanagi-Harada disease. J Immunol 165: 7323-7329.

19. Chan CC, Palestine AG, Nussenblatt RB, Roberge FG, Benezra D (1985) Anti-retinal auto-antibodies in Vogt-Koyanagi-Harada syndrome, Behcet's disease, and sympathetic ophthalmia. Ophthalmology 92: 1025-1028.

20. Yamaki K, Takiyama N, Itho N, Mizuki N, Seiya M, et al. (2005) Experimentally induced Vogt-Koyanagi-Harada disease in two Akita dogs. Exp Eye Res 80: 273-280.

21. Otani S, Sakurai T, Yamamoto K, Fujita T, Matsuzaki Y, et al. (2006) Frequent immune response to a melanocyte specific protein KU-MEL-1 in patients with Vogt-Koyanagi-Harada disease. Br J Ophthalmol 90: 773-777.

22. Wetts R, Fraser SE (1988) Multipotent precursors can give rise to all major cell types of the frog retina. Science 239: 1142-1145.

23. Perron M, Harris WA (2000) Retinal stem cells in vertebrates. Bioessays 22: 685-688.

24. Ahmad I, Tang L, Pham H (2000) Identification of neural progenitors in the adult mammalian eye. Biochem Biophys Res Commun 270: 517-521.

25. Tropepe V, Coles BL, Chiasson BJ, Horsford DJ, Elia AJ, et al. (2000) Retinal stem cells in the adult mammalian eye. Science 287: 2032-2036.

26. Frøen R, Johnsen EO, Nicolaissen B, Facskó A, Petrovski G, et al. (2013) Does the adult human ciliary body epithelium contain "true" retinal stem cells? Biomed Res Int 2013: 531579.
27. Ballios BG, Clarke L, Coles BL, Shoichet MS, Van Der Kooy D (2012) The adult retinal stem cell is a rare cell in the ciliary epithelium whose progeny can differentiate into photoreceptors. Biol Open 1: 237-246.

28. Xu H, Sta Iglesia DD, Kielczewski JL, Valenta DF, Pease ME, et al. (2007) Characteristics of progenitor cells derived from adult ciliary body in mouse, rat, and human eyes. Invest Ophthalmol Vis Sci 48: 1674-1682.

29. Das AV, James J, Zhao X, Rahnenführer J, Ahmad I (2004) Identification of c-Kit receptor as a regulator of adult neural stem cells in the mammalian eye: interactions with Notch signaling. Dev Biol 273: 87-105.

30. Le Douarin, N,Kalcheim C (1999) The Neural Crest. Cambridge University Press, Cambridge, UK.

31. Le Lièvre CS, Le Douarin NM (1975) Mesenchymal derivatives of the neural crest: analysis of chimaeric quail and chick embryos. J Embryol Exp Morphol 34: 125-154.

32. Takahashi K, Tanabe K, Ohnuki M, Narita M, Ichisaka T, et al. (2007) Induction of pluripotent stem cells from adult human fibroblasts by defined factors. Cell 131: 861-872.

33. Yu J, Vodyanik MA, Smuga-Otto K, Antosiewicz-Bourget J, Frane $\mathrm{JL}$, et al. (2007) Induced pluripotent stem cell lines derived from human somatic cells. Science 318: 1917-1920. 